\title{
VITCOMIC2: visualization tool for the phylogenetic composition of microbial communities based on 16S rRNA gene amplicons and metagenomic shotgun sequencing
}

\author{
Hiroshi Mori ${ }^{1 *}$, Takayuki Maruyama², Masahiro Yano², Takuji Yamada ${ }^{2}$ and Ken Kurokawa ${ }^{1}$
}

From The 28th International Conference on Genome Informatics

Seoul, Korea. 31 October - 3 November 2017

\begin{abstract}
Background: The 16S rRNA gene-based amplicon sequencing analysis is widely used to determine the taxonomic composition of microbial communities. Once the taxonomic composition of each community is obtained, evolutionary relationships among taxa are inferred by a phylogenetic tree. Thus, the combined representation of taxonomic composition and phylogenetic relationships among taxa is a powerful method for understanding microbial community structure; however, applying phylogenetic tree-based representation with information on the abundance of thousands or more taxa in each community is a difficult task. For this purpose, we previously developed the tool VITCOMIC (VIsualization tool for Taxonomic COmpositions of Mlcrobial Community), which is based on the genome-sequenced microbes' phylogenetic information. Here, we introduce VITCOMIC2, which incorporates substantive improvements over VITCOMIC that were necessary to address several issues associated with $16 \mathrm{~S}$ rRNA gene-based analysis of microbial communities.

Results: We developed VITCOMIC2 to provide (i) sequence identity searches against broad reference taxa including uncultured taxa; (ii) normalization of 16S rRNA gene copy number differences among taxa; (iii) rapid sequence identity searches by applying the graphics processing unit-based sequence identity search tool CLAST; (iv) accurate taxonomic composition inference and nearly full-length $16 \mathrm{~S}$ rRNA gene sequence reconstructions for metagenomic shotgun sequencing; and ( $v$ ) an interactive user interface for simultaneous representation of the taxonomic composition of microbial communities and phylogenetic relationships among taxa. We validated the accuracy of processes (ii) and (iv) by using metagenomic shotgun sequencing data from a mock microbial community.

Conclusions: The improvements incorporated into VITCOMIC2 enable users to acquire an intuitive understanding of microbial community composition based on the 16S rRNA gene sequence data obtained from both metagenomic shotgun and amplicon sequencing.
\end{abstract}

Keywords: $16 \mathrm{~S}$ rRNA gene, Microbial community, Metagenomics, Taxonomic composition

\footnotetext{
* Correspondence: hmori@nig.ac.jp

'Genome Evolution Laboratory, Center for Information Biology, National

Institute of Genetics, Mishima 411-8540, Japan

Full list of author information is available at the end of the article
} 


\section{Background}

Sequence analysis of the 16S rRNA gene-based amplicon is widely used to determine structures of microbial communities $[1,2]$. To understand such structures from a phylogenetic viewpoint, it is helpful to have a combined representation of the taxonomic composition of microbial communities and the phylogenetic relationships among taxa [3, 4]. These relationships can be represented by a phylogenetic tree, yet abundance information on thousands or more taxa in the community is difficult to represent simultaneously in a tree $[5,6]$. To address this issue, we previously developed the tool VITCOMIC (VIsualization tool for Taxonomic COmpositions of MIcrobial Community) [7, 8]. VITCOMIC compares userprovided 16S rRNA gene sequences with reference $16 \mathrm{~S}$ rRNA gene sequences obtained from genome-sequenced microbes and identifies the nearest relative of each submitted sequence. VITCOMIC then renders a visualization of the overall taxonomic composition of the sample with indications of sequence identity between the reference and sample sequences. Because VITCOMIC yields a combined representation of the taxonomic composition of a sample and the phylogenetic relationships among taxa, this tool has been adopted by many researchers [9-11].

Substantive improvements in VITCOMIC became necessary to address several issues related to the analysis of $16 \mathrm{~S}$ rRNA gene-based microbial communities, noted as follows. (i) Public databases contain many $16 \mathrm{~S}$ rRNA gene sequences from uncultured taxa [12], but VITCOMIC uses $16 \mathrm{~S}$ rRNA gene sequences exclusively from genomesequenced prokaryotes as references. (ii) The genomes of most microbes contain two or more copies of $16 \mathrm{~S}$ rRNA genes $[13,14]$, but VITCOMIC does not normalize the bias of this taxonomic abundance that results from differences in $16 \mathrm{~S}$ rRNA gene copy number among taxa. (iii) The number of sequences for each sample has increased significantly because of the rapid technological developments related to massively parallel DNA sequencing [15], but the calculation speed of VITCOMIC has not kept pace with data growth because VITCOMIC uses BLAST [16] for sequence identity searches. (iv) The use of $16 \mathrm{~S}$ rRNA gene sequences obtained by shotgun metagenomic sequencing eliminates any bias that might result from the initial PCR amplification of $16 \mathrm{~S}$ rRNA genes $[17,18]$, but the extraction of $16 \mathrm{~S}$ rRNA gene sequences from such sequencing data based on sequence similarity searches against reference databases is problematic because some $16 \mathrm{~S}$ rRNA gene sequences in public databases are contaminated by sequences from other genes (e.g., tRNA and 23S rRNA genes) [18]. To address these issues, we developed VITCOMIC2, which enables users to acquire an intuitive understanding of microbial community composition based on sequence data for the 16S rRNA gene obtained from both metagenomic shotgun and amplicon sequencing.

\section{Implementation}

Construction of a high-quality 16S rRNA gene sequence reference database including uncultured taxa

A high-quality $16 \mathrm{~S}$ rRNA gene sequence reference database that includes uncultured taxa was constructed using 16S rRNA gene sequences obtained from the Ribosomal Database Project (RDP) [12]. The 1,345,732 16S rRNA gene sequences of Bacteria and Archaea in RDP (release 11, update 2) were retrieved using the following parameters: Strain $=$ Both, Source $=$ Both, Size $\geq 1200$, and Quality $=$ Good. We obtained these sequences with three different file formats: multiply aligned fasta files, unaligned fasta files, and GenBank files. To eliminate contaminating tRNA gene sequences, internal transcribed spacer sequences (ITSs), and 23S rRNA gene sequences from this reference database, we performed a search of the 1,345,732 sequences with BLAST+ [19] (version 2.2.27; -max_target_seqs 100 and -parc_identity 97) against the previous VITCOMIC 16S rRNA gene sequence reference database [20]. This previous database contains only $16 \mathrm{~S}$ rRNA gene sequences for which the 5 '-end was adjusted to six bases before the $8 \mathrm{~F}$ primer motif sequence (TTGATCCT) [21] and the 3 '-end, adjusted to the end of the anti-Shine-Dalgarno sequence (CACCTCCTTN) [22]; therefore, the $5^{\prime}$ - and $3^{\prime}$-end trimming of each sequence for optimization of VITCOMIC2 was easily performed using the sequence identity search results. After trimming the $1,345,73216 \mathrm{~S}$ rRNA gene sequences, we eliminated (i) 476 sequences for which the length was abnormally long (> $1660 \mathrm{bp}$ ) or short (<1120 bp) according to the distribution of sequence length (Additional file 1), and (ii) 1159 sequences that contained $\geq 10$ bases of homopolymer.

Because the RDP database contains many nearly identical sequences, we performed genus-level sequence clustering using USEARCH [23] version 6.0.307 (--cluster_smallmem -id 0.94, --query_cov 0.9, and -target_cov 0.9). Since USEARCH/UCLUST version 6 uses a greedy algorithm, the order of input sequences is very important [23]. Therefore, before conducting sequence clustering, we have sorted sequences whose origins at the genus-level taxonomy were not designated as "unclassified" by their sequence lengths. Then, "unclassified" sequences were sorted by their sequence lengths, and added on the end of the sequence file. By sorting sequences as described above, in most cases, we can avoid the "unclassified" sequences in the RDP database become a representative sequence of a cluster. The resulting 63,956 representative sequences of clusters were examined to determine whether any were PCR chimeric sequences using UCHIME [24] (version 6.0.307 de novo mode and reference mode). In the UCHIME reference mode, gold.fa sequence data [25] were used as the reference. We discarded the 706 representative sequences 
that were flagged by both the de novo and reference modes of UCHIME as chimeric sequences. In addition, we discarded the 34,273 representative sequences whose origins at the genus-level taxonomy were designated as "unclassified". Consequently, we obtained a database that consisted of 28,977 high-quality $16 \mathrm{~S}$ rRNA gene sequences (Additional file 2 and Additional file 3).

\section{Allocation of references in VITCOMIC2}

In the previous version of VITCOMIC, VITCOMIC visualized overall phylogenetic composition of a sample in one circular diagram using $16 \mathrm{~S}$ rRNA gene sequences of genome sequenced prokaryote species as reference [7]. However, the number of genome sequenced prokaryote species are rapidly grown. In addition, the uncultured taxa whose genomes are not sequenced yet are also important to describe microbial communities. To include many reference sequences in VITCOMIC2, we need to separate a VITCOMIC circular diagram to two types of circular diagrams representing overall phylogenetic composition of a microbial community based on phylum composition and detailed phylogenetic composition in each phylum of the community based on genus composition. Since the separation of phylum and genus composition in different circular diagrams often cause difficulty to understand overall phylogenetic composition of a microbial community, we completely replaced a Perl and PostScript based static circular diagram in VITCOMIC to JavaScript Raphael library based interactive circular diagrams in VITCOMIC2.

VITCOMIC2 constructs a circular diagram representing overall phylogenetic composition of a microbial community based on phylum composition with the following procedure. (i) In each phylum, 30 sequences were randomly chosen from the high-quality 16S rRNA gene sequence database. As the phylum Proteobacteria is extraordinarily diverse [26], we randomly chose 30 sequences from every class of Proteobacteria. For any phylum having $<30$ sequences, all available sequences were chosen. (ii) We thus obtained 1184 sequences from 44 phyla/classes. To calculate phylogenetic distances among them, the 1184 sequences were aligned using MAFFT [27] version $6.864 \mathrm{~b}$ with default parameters. After constructing a multiple sequence alignment, genetic distances between sequences were calculated using the dnadist program in PHYLIP [28] version 3.69 with Kimura's two-parameter model of base substitution [29]. The phylogenetic tree was constructed using the neighbor-joining method in the neighbor program of PHYLIP version 3.69. (iii) To construct the circular diagram from the phylogenetic tree, we randomly chose one sequence from Gammaproteobacteria to use as the initial sequence for subsequent tree topology scanning. The tree topology scanning is our concise method to represent tree topology (distance and cladogram) information in one line as follows. The sequence that was most distantly related to the initial sequence was identified by comparing the phylogenetic distances between the initial sequence and every other sequence (Fig. 1). The second most distantly related sequence was identified in the peripheral clade that contained the most unrelated sequence, and this process was reiterated to generate an ordered list of related sequences. (iv) The position on the circular diagram of each sequence in the list was determined based on its phylogenetic distance to its nearest relative. (v) Each phylum/class position in the circular diagram was determined by calculating an average position among sequences that belong to that phylum/class.

To understand the detailed taxonomic composition in each phylum, VITCOMIC2 also constructs a circular diagram that represents the genus composition of the sample as described by the following example. (i) The phylogenetic tree within each of the 44 phyla/classes was constructed by using all sequences belonging to each phylum/class in the high-quality $16 \mathrm{~S}$ rRNA gene sequence database, i.e., the same method that was used for the phylum example. (ii) The same method was again used to construct the circular diagram within each of the 44 phyla/classes with a random choice of one sequence to serve as the initial sequence. If all the sequences derived from the same genus belonged to a single cluster, then the genus position in the circular diagram was the cluster position. Conversely, when the sequences derived from the same genus belonged to more than two clusters, the genus position in the circular diagram was determined by calculating an average position among the clusters.

\section{A rapid sequence identity search using graphics processing units (GPUs)}

The overall workflow of VITCOMIC2 for processing data from user-uploaded 16S rRNA gene amplicon sequencing and metagenomic shotgun sequencing is described in Fig. 2. The inference of the nearest relative for each query sequence in VITCOMIC2 was performed with a modified version of CLAST [30], a GPU-based high-speed nucleotide sequence identity search tool that we developed. We made the following modifications to CLAST to enable us to specifically search $16 \mathrm{~S}$ rRNA gene sequences. (i) We completely separated the processes of database indexing and alignment calculation. Because VITCOMIC2 does not update the reference sequence database frequently, pre-indexing the reference database is more efficient for daily calculations. (ii) We changed the method used to estimate the amount of GPU memory consumed. In the original CLAST program, total nucleotide lengths of sequences were used to estimate the amount of the GPU memory consumed. In the modified version of CLAST for VITCOMIC2, referred to as CLASTV, 


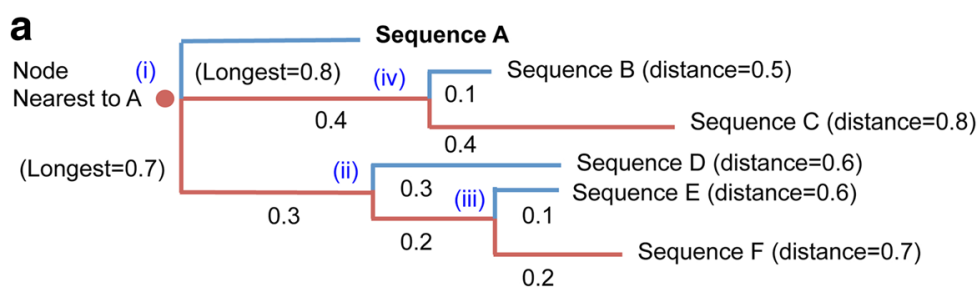

b

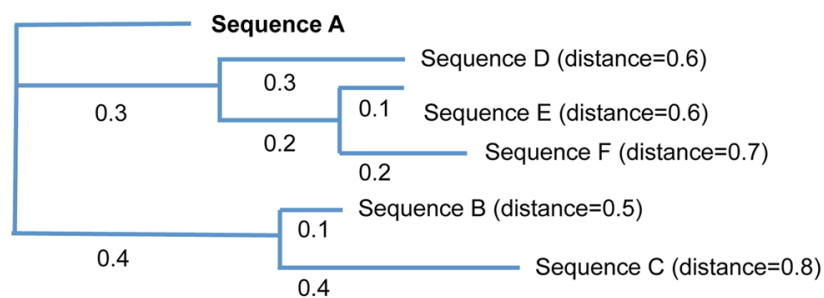

C

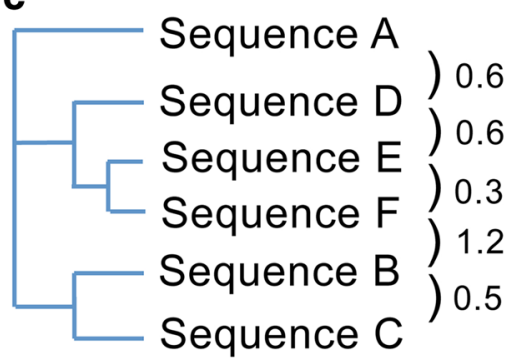

Fig. 1 Schematic diagram of the method used to determine the order of sequences in a circular diagram. (a) The original phylogenetic tree that contains six sequences. Sequence A represents the initial sequence chosen for subsequent tree topology scanning. The order of the six sequences in the phylogenetic tree was determined as follows. Relative to sequence $A$, sequence $C$ is the farthest, sequence $B$ is the second farthest, sequence $F$ is the third farthest, sequence $\mathrm{E}$ is the fourth farthest, and sequence $\mathrm{D}$ is nearest. (b) A rotated phylogenetic tree based on the order of sequences determined as in (a). (c) The order of, and phylogenetic distances between, the sequences that were determined as in (a)

the total number of sequences was used because there was little variation in the lengths of the query or database sequences. We compared the calculation speed between VITCOMIC2 and VITCOMIC by using 1,119,519 16S rRNA gene sequences sequenced by the 454 GS FLX sequencing platform obtained from Turnbaugh et al. [31] as query sequences. For VITCOMIC we used one process of BLAST+ with the parameters (-evalue 1e-08 and -num_alignments 100 ) and for VITCOMIC2 we used one process of CLASTV with the thresholds (identity $\geq 80 \%$ and alignment length $\geq$ $50 \mathrm{bp})$. The speed test was carried out on a machine with a Xeon X5670 2.93 GHz CPU and a Tesla C2050 GPU.

\section{Normalizing differences in 16S rRNA gene copy number among taxa}

Although some microbial genomes contain only one copy of the $16 \mathrm{~S}$ rRNA gene, most microbes contain two or more copies [13]. Therefore, the taxonomic abundance of a microbial community directly inferred from $16 \mathrm{~S}$ rRNA gene sequence abundance is generally biased because of differences in 16S rRNA gene copy number among taxa [32]. To avoid this bias in VITCOMIC2, we implemented the following method to normalize $16 \mathrm{~S}$ rRNA gene copy number differences among taxa. Previous work suggested that phylogenetically cross-related taxa tend to have a similar number of $16 \mathrm{~S}$ rRNA gene copies in the genome [33]. To obtain 16S rRNA gene copy number information for the phylogenetically related taxa of the user sequence, VITCOMIC2 conducts a sequence identity search using CLASTV against a $16 \mathrm{~S}$ rRNA gene copy number reference database (16S rRNA$\mathrm{CN} \mathrm{DB}$ ) of the genome-sequenced strains, which was constructed as follows. (i) The $16 \mathrm{~S}$ rRNA gene sequences for each genome-sequenced strain were identified using RNAmmer [34] against the genomic sequence obtained from the NCBI Genome Database [35] in April 2014. (ii) One $16 \mathrm{~S}$ rRNA gene sequence was randomly sampled per species because there are only small sequence differences among 16S rRNA genes within a single genome and across genomes within a single species [13]. We obtained 1505 16S rRNA gene sequences, which thus comprised the $16 \mathrm{~S}$ rRNA-CN DB (Additional file 4). The sequence most related to the user sequence was then identified based on the results of a sequence identity 


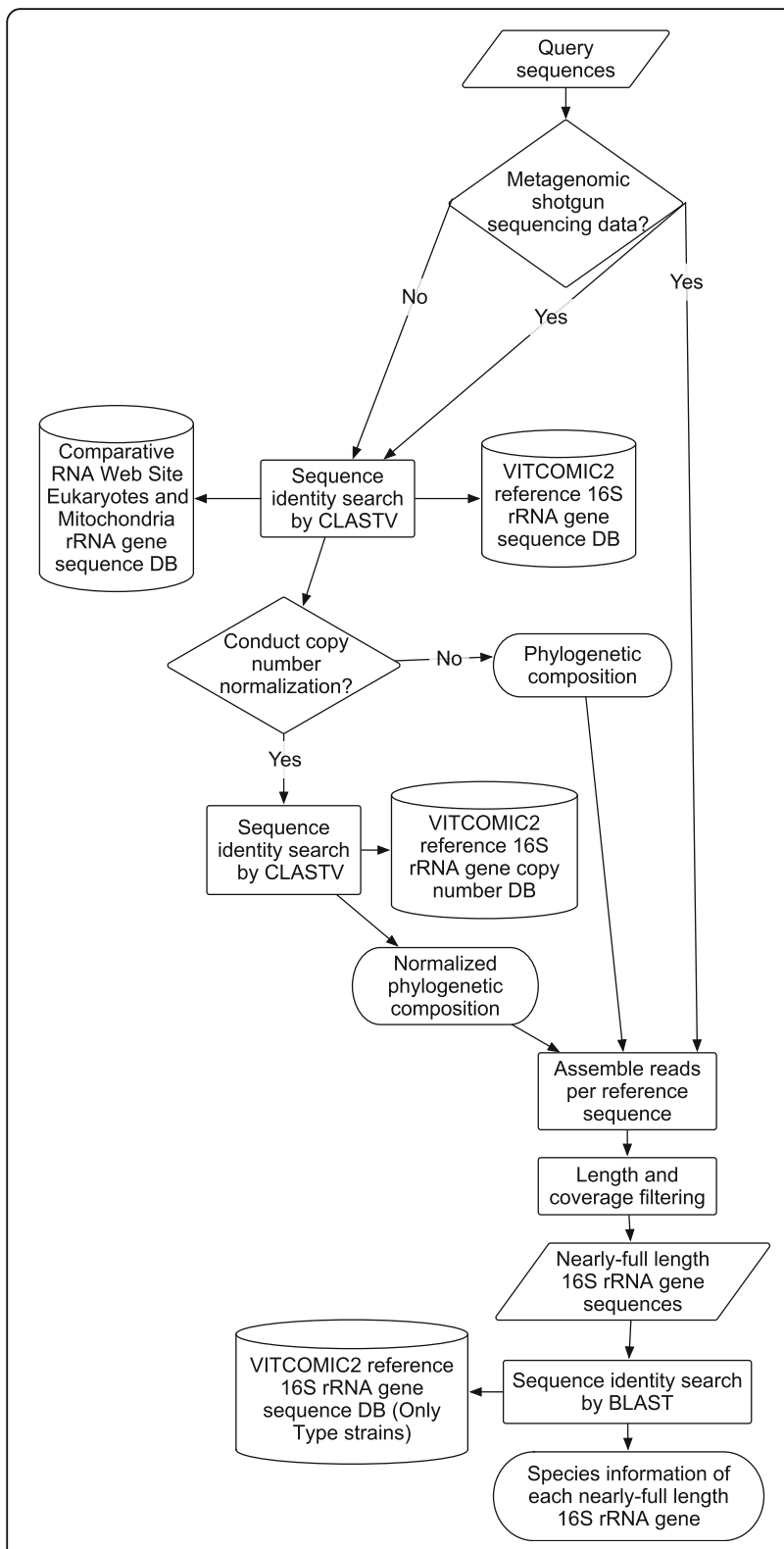

Fig. 2 VITCOMIC2 overall workflow. The workflow is shown with the following symbols: parallelogram, sequence data; cylinder, sequence database; square, analysis; diamond, conditional branch; ellipse, output file

search against the $16 \mathrm{~S}$ rRNA-CN DB. VITCOMIC2 assumes that the 16S rRNA gene copy number is identical between the genomes of the user sequence and the nearest sequence in the $16 \mathrm{~S}$ rRNA-CN DB.

We used a leave-one-out approach to validate the accuracy of the VITCOMIC2 16S rRNA gene copy number inference method. To identify the most related sequence, we randomly chose one sequence from the $16 \mathrm{~S}$ rRNA$\mathrm{CN} \mathrm{DB}$ and conducted a sequence identity search with BLAST + (-max_target_seqs 100) against this database (lacking the query sequence). Comparing the 16S rRNA gene copy number between the genomes of the query sequence and the nearest sequence, we calculated the error ratio for inferring the 16S rRNA gene copy number for all 1505 sequences from the 16S rRNA-CN DB.

\section{Taxonomic composition analysis using the 16S rRNA gene sequences extracted from metagenomic shotgun sequencing data}

To evaluate the specificity of the VITCOMIC2 extraction method for $16 \mathrm{~S}$ rRNA gene sequences from metagenomic shotgun sequencing data, we compared the VITCOMIC2 method with a common extraction method using BLAST + against the original RDP sequence database. Because we needed to eliminate the effect of different sensitivities between the two databases (i.e., the effect of different taxonomic coverage between the two databases on this comparison), we designated the $1,345,256$ sequences in the VITCOMIC2 16S rRNA gene sequence reference database before conducting homopolymer filtering and 94\% identity clustering as the reference sequence database. For comparison, we used soil metagenomic shotgun sequencing data (DRR001464) [18], as sequenced by the Illumina Genome Analyzer IIx sequencer. For the alternative sequence extraction method, the 47,195,934 sequences from DRR001464 were searched against the original RDP sequence database using BLAST+ with Evalue $<1 \mathrm{e}-8$. The sequences extracted from the VITCOMIC2-based method and the original RDP sequence database-based method were searched against the NCBI-nt database [36] obtained as of July 2014 using BLAST + with E-value <1e-4; each hit was manually verified with regard to gene function to evaluate whether the specific extracted sequence was indeed a 16S rRNA gene.

\section{Validation of accuracy of the genus assignments and the} 16S rRNA gene copy number normalization by using mock community metagenomic data

We used the Illumina HiSeq metagenomic sequencing data of Singer et al. [37] (read length $=150 \mathrm{bp}$ ) of a mock community of 26 genome-sequenced strains (23 genera) for the validation of accuracy of the genus assignments and the 16S rRNA gene copy number normalization.

The molarity of the DNA of each species in the mock community was previously measured [37]. In addition, the 16S rRNA gene copy number in the genome of each species is described in the RefSeq genome sequence database [36]. Therefore, the theoretical abundance of $16 \mathrm{~S}$ rRNA gene sequences from each species in the mock community metagenomic sequencing data was calculated by multiplying the molarity of the DNA of each species and the 16S rRNA gene copy number in the genome. We compared the genus compositions of communities resulting from (i) the theoretical 
composition based on the molarity of the DNA of each species, (ii) the theoretical composition based on multiplying the molarity of the DNA of each species and the $16 \mathrm{~S}$ rRNA gene copy number of the genome, (iii) $16 \mathrm{~S}$ rRNA gene sequence-based composition calculated by VITCOMIC2, and (iv) 16S rRNA gene sequence-based composition calculated by VITCOMIC2 with $16 \mathrm{~S}$ rRNA gene copy number normalization.

In addition, we compared genus composition inference accuracies of the Singer et al. mock community sequencing data among VITCOMIC2 and three other software (MAPSeq version 1.2 [38], SortMeRNA version 2.1b [39], and RiboTagger version 0.8.1 [40]). To separate the reference sequence databases differences and the genus composition inference accuracies of tools, we used the VITCOMIC2 high-quality $16 \mathrm{~S}$ rRNA gene reference sequence database as a reference sequence database for MAPSeq and SortMeRNA. In the VITCOMIC2, MAPSeq, and SortMeRNA results, we only used hits with the threshold (identity $\geq 94 \%$, alignment length $\geq 100 \mathrm{bp}$, and top hit) for genus assignments. In the RiboTagger analysis, we specified a parameter "-r v4".
Species inference based on the nearly full-length $16 \mathrm{~S}$ rRNA gene sequence reconstructed from metagenomic shotgun sequencing data

The ecological niches of taxa are sometimes different among species in the same genera [41]. However, the partial 16S rRNA gene sequences obtained from the metagenomic or amplicon sequencing analyses by the short read sequencers usually do not contain enough information for species identification [42]. Therefore, we developed a function to reconstruct nearly full-length 16S rRNA gene sequences from metagenomic shotgun sequencing data in VITCOMIC2. The reconstruction workflow is as follows. (i) Based on CLASTV results in VITCOMIC2, 16S rRNA gene reads from metagenomic shotgun sequencing data that are assigned to the same reference sequence are extracted and assembled by MEGAHIT version 1.1.1 with default parameters. (ii) After contig length $(\geq 800 \mathrm{bp})$ and read coverage $(\geq 10)$ filtering, nearly full-length 16S rRNA gene sequences are obtained. (iii) BLAST-based sequence identity searches are conducted using the nearly full-length $16 \mathrm{~S}$ rRNA gene sequences against the type strain 16S rRNA gene sequences in the VITCOMIC2 16S rRNA gene sequence

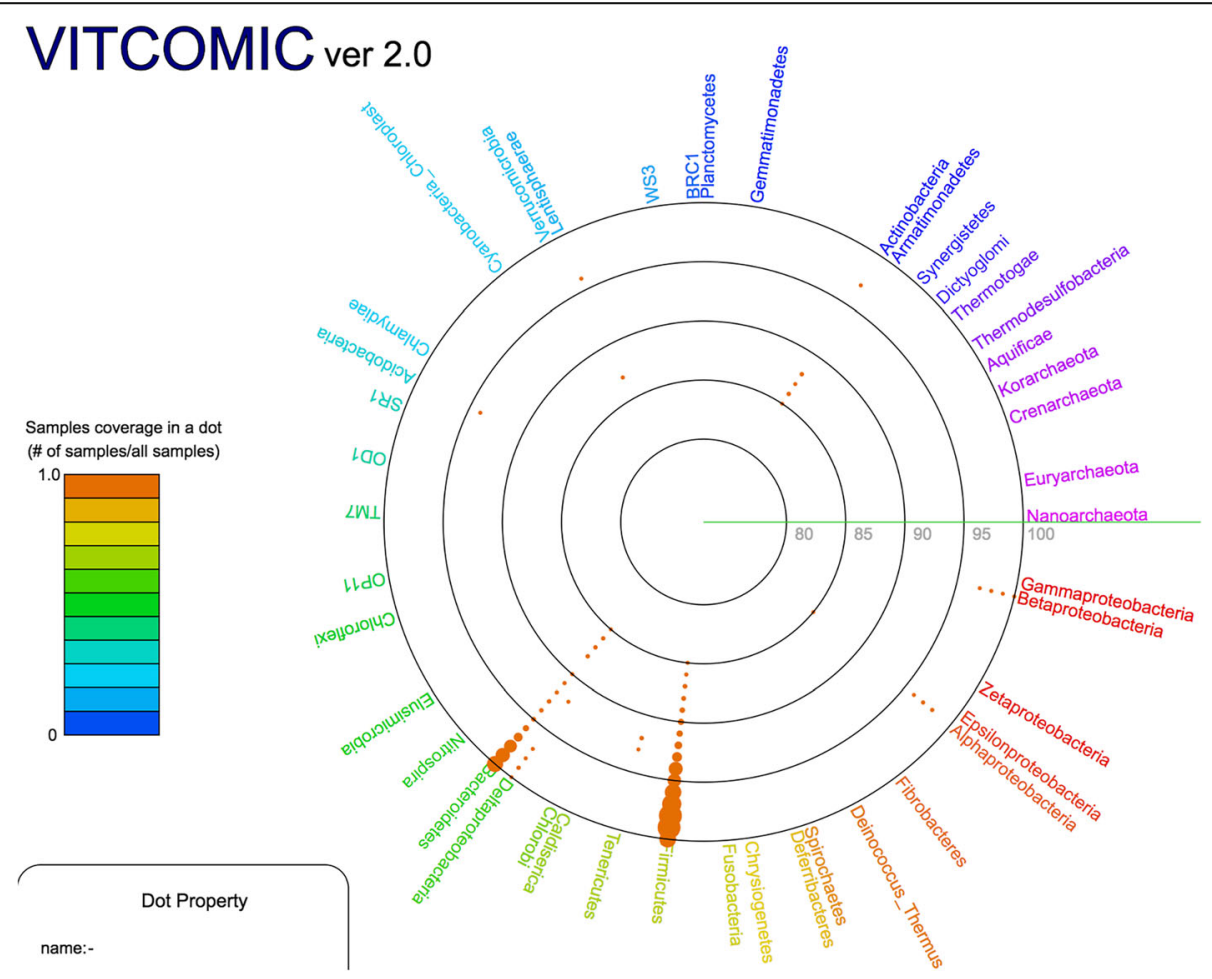

Fig. 3 Example of VITCOMIC2 representation of the phylum composition and associated phylogenetic relationships. Phylum composition of a sample from the Turnbaugh et al. study [31]. For VITCOMIC2, the basic representation strategy does not differ from that of the original VITCOMIC, although the following additional information is represented in VITCOMIC2. (i) Sizes of dots represent the relative abundance of reads in the sequence cluster. (ii) When users select a dot, they obtain information on the number of sequences that the cluster contains. (iii) Genus composition of the phylum can be viewed by selecting the phylum name 
reference database. (iv) The names of species are assigned to the nearly full-length $16 \mathrm{~S}$ rRNA gene sequences when $\geq 97 \%$ sequence identities are observed in the BLAST-based sequence identity searches from (iii). The accuracy of the above species inference method was evaluated by using Illumina HiSeq metagenomic data of the mock community [37].

\section{VITCOMIC2 web system}

The VITCOMIC2 web server [43] can accept either a fasta or a fastq file of one $16 \mathrm{~S}$ amplicon or metagenomic shotgun sequencing sample with read length $\geq 100 \mathrm{bp}$ as the query. Users can choose whether to include $16 \mathrm{~S}$ rRNA gene copy number normalization and nearly fulllength 16S rRNA gene sequence reconstruction. After uploading an input file, VITCOMIC2 conducts analyses as described in Fig. 2. (i) VITCOMIC2 conducts a CLASTV-based sequence identity search against the VITCOMIC2 high-quality 16S rRNA gene reference sequence database with the threshold (identity $\geq 80 \%$ and alignment length $\geq 100 \mathrm{bp}$, and top hit). (ii) To exclude rRNA gene sequences of eukaryotes and mitochondria, VITCOMIC2 also conducts CLASTV-based sequence identity searches against the $18 \mathrm{~S}$ rRNA gene and rRNA gene sequences of eukaryotes and mitochondria obtained from Comparative RNA Web Site version 2 [44] with the same threshold. (iii) If users didn't choose to conduct 16S rRNA gene copy number normalizations and the nearly full-length $16 \mathrm{~S}$ rRNA gene sequence reconstructions, a genus composition tab-separated text file by setting identity threshold $\geq 94 \%$ and VITCOMIC 2 phylum and genus composition figures are created by using information of CLASTV hits which were in (i)'s analysis but were not in (ii)'s analysis. (iv) In case of conducting 16S rRNA gene copy number normalizations, VITCOMIC2 conducts a CLASTV-based sequence identity search against the VITCOMIC2 $16 \mathrm{~S}$ rRNA-CN DB after (i) and (ii) analyses. Using the VITCOMIC2 $16 \mathrm{~S}$ rRNA-CN DB information, each hit in (i)'s analysis is normalized as described above section. After that, a normalized genus composition tab-separated text file and VITCOMIC2 phylum and genus composition figures are created. (v) In case of conducting nearly full-length $16 \mathrm{~S}$ rRNA gene sequence reconstructions from metagenomic shotgun sequencing data, MEGAHIT assembling and BLAST-based sequence identity search against the type strain 16S rRNA gene sequences in VITCOMIC2 reference sequence database are performed as described above section. When the VITCOMIC2 analysis is finished, VITCOMIC2 sends an Email to the user with a download link to the results which include a VITCOMIC2 visualization result, a genus composition text file,

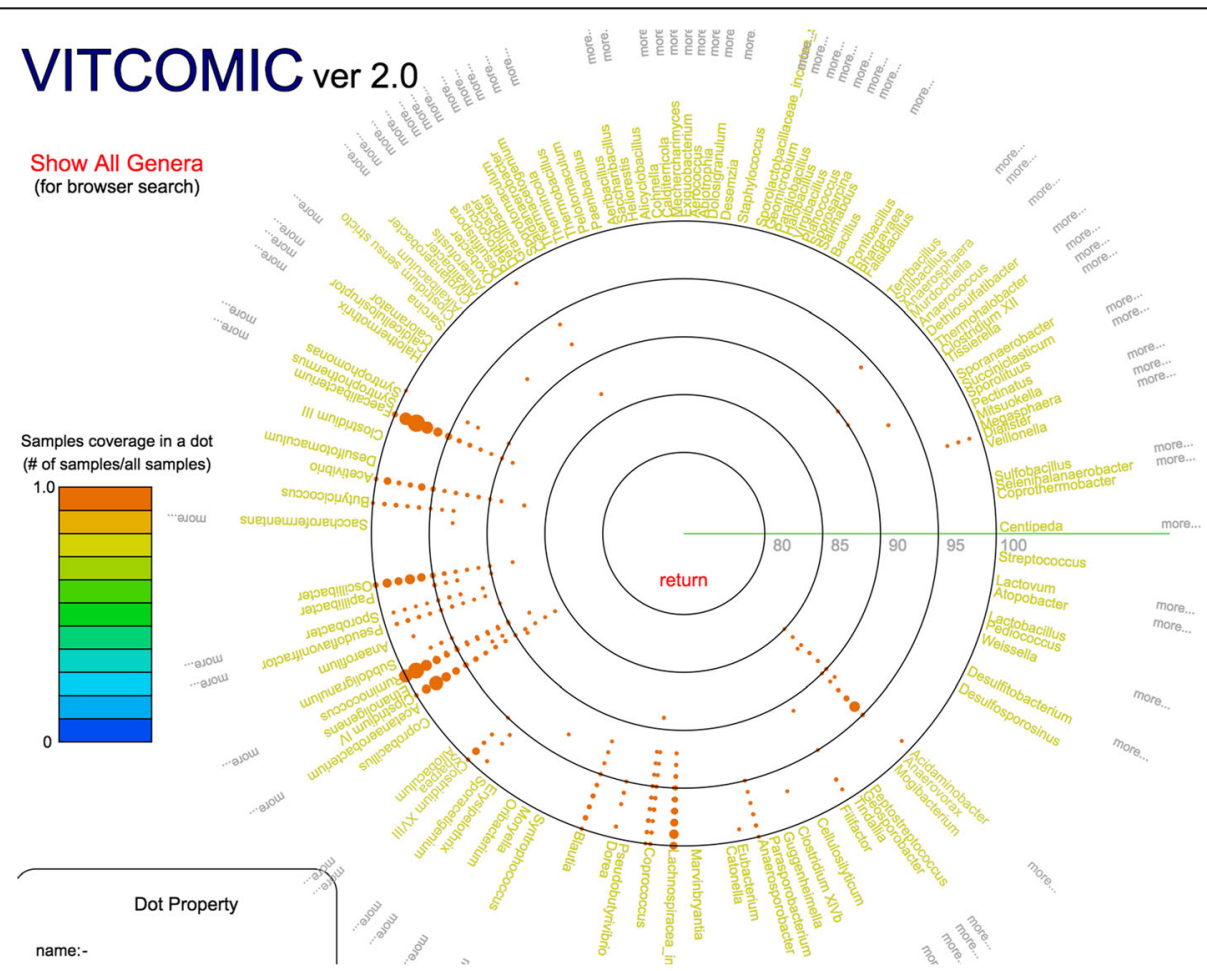

Fig. 4 Example of VITCOMIC2 representation of the genus composition and associated phylogenetic relationships. Genus composition of a samples from the Turnbaugh et al. study [31]. When two or more genera are located at the same location, these genera names can be seen by selecting the "more" button for specific genera, or by selecting the "Show All Genera" button to view all genera at once 
reconstructed nearly full-length $16 \mathrm{~S}$ rRNA gene sequences fasta files and species assignment result text files, and a text file for the VITCOMIC2 Comparison. Users can conduct multiple-sample comparisons by uploading VITCOMIC2 analysis result files for selected samples to the VITCOMIC2 Comparison web server [45].

\section{Results and discussion}

\section{Description of the VITCOMIC2 circular diagram}

VITCOMIC2 represents the phylum and genus composition of samples and their phylogenetic relationships using a circular diagram (Fig. 3 and Fig. 4). VITCOMIC2 Comparison calculates several statistical coefficients for pairwise community comparison (Jaccard similarity, Pearson correlation, and Yue and Clayton theta similarity coefficients [7]) (Fig. 5). In VITCOMIC2 Comparison, to conduct an accurate statistical comparison between samples, only those query sequences that match a reference database sequence with $\geq 94 \%$ identity are used. These features of the VITCOMIC2 circular diagram help the user understand microbial community composition more intuitively.

In the previous version of VITCOMIC, to fit the order of reference taxa on the VITCOMIC circular diagram with the known phylogenetic relationships among taxa, we had to manually rotate the reference phylogenetic tree. For a phylogenetic tree with 28,977 branches, this rotation was time consuming. In addition, we have little knowledge of the phylogenetic relationships for the uncultured taxa. Therefore, we developed a reference taxa allocation method to construct the VITCOMIC2 circular diagram automatically. Application of this method to the previous VITCOMIC reference data confirmed that the order of the species remained the same as that determined with the manual rotation-based circular diagram (Spearman rank correlation coefficient $=0.98$ ), thereby validating the VITCOMIC2 reference taxa allocation method.

\section{Sensitivity and speed of VITCOMIC2 as compared with VITCOMIC}

The time needed to analyze the 1,119,519 16S rRNA gene amplicon sequences [31] using VITCOMIC2 was $20 \mathrm{~min}$, much faster than the $5 \mathrm{~h} 31$ min needed for VITCOMIC. This vast improvement in speed was achieved despite the fact that VITCOMIC only contains only $60116 \mathrm{~S}$ rRNA gene reference sequences and no normalization calculation function for determining the $16 \mathrm{~S}$ rRNA gene copy number. The number of reads whose identity with the reference sequences was $\geq 80 \%$ was 1,106,215 for VITCOMIC and 1,112,958 for VITCOMIC2. The average top hit identity of these reads was $92.3 \%$ for VITCOMIC and $98.0 \%$ for VITCOMIC2. For the taxonomic composition analysis based on $16 \mathrm{~S}$ rRNA gene sequences, unassigned sequences are usually neglected for the analysis, and this is especially the case

\section{VITCOMIC ver 2.0}

Mode: Yue \& Clayton

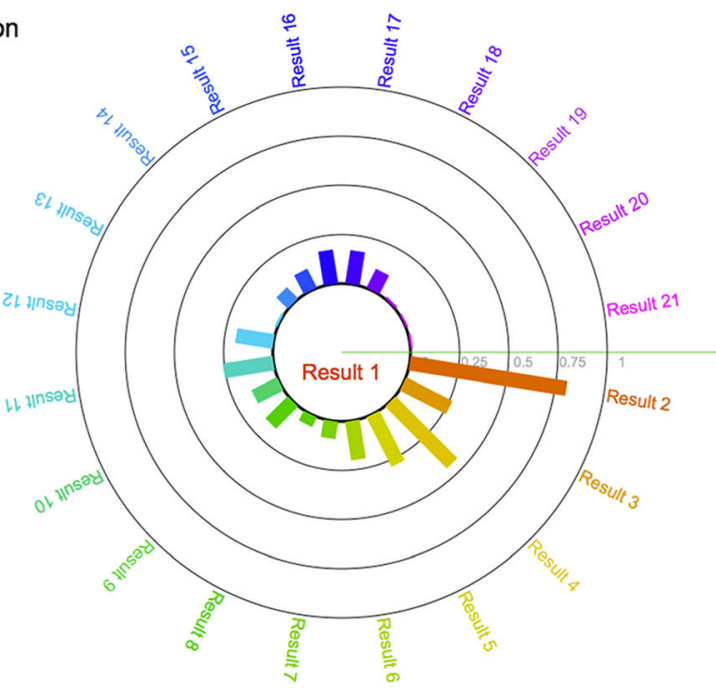

曾

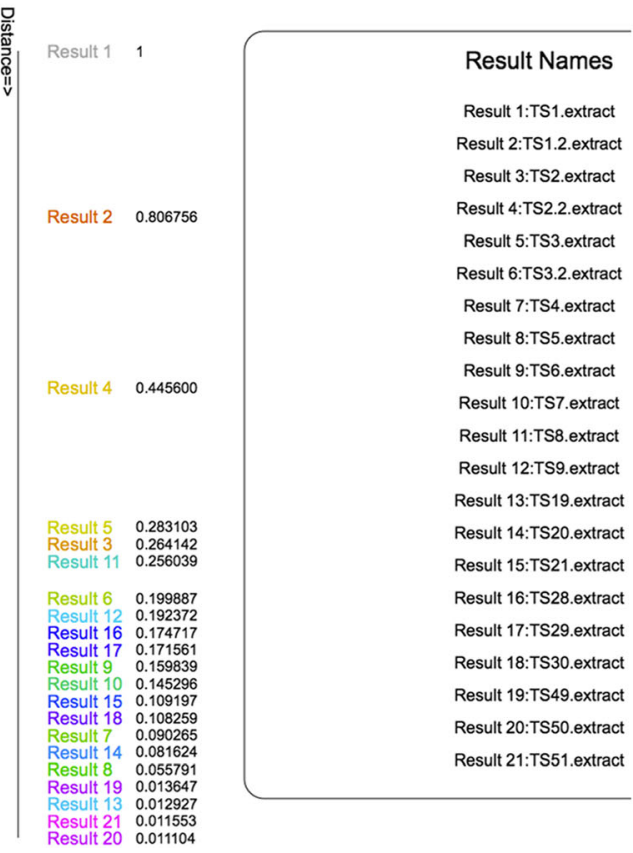

Fig. 5 Example of a VITCOMIC2 statistical sample comparison. The sequence cluster composition among samples is used to calculate Jaccard similarity, Pearson correlation, and the Yue and Clayton theta similarity coefficients. The Yue and Clayton theta similarity coefficient between a particular sample and the other 21 samples from the Turnbaugh et al. study [31] is shown. Users can change the sample for comparison by selecting the sample name 
for metagenomic shotgun sequencing, for which it is difficult to confirm that the unassigned sequences are definitely not $16 \mathrm{~S}$ rRNA genes. Therefore, it is crucial to maximize the sensitivity of the taxonomy assignment and reduce the fraction of unassigned sequences so that the taxonomic composition of microbial communities can be appropriately inferred. To maintain sensitivity but reduce calculation time, we removed phylogenetically redundant sequences in the reference database by conducting sequence clustering. Using CLASTV and the phylogenetically non-redundant high-quality $16 \mathrm{~S}$ rRNA gene sequence database including uncultured taxa, VITCOMIC2 greatly improved the sensitivity and reduced the calculation time as compared with VITCOMIC.

\section{Validation of the VITCOMIC2 16S rRNA gene copy number normalization method}

Additional file 5 presents the results of the leave-one-out accuracy validation for the VITCOMIC2 normalization method for $16 \mathrm{~S}$ rRNA gene copy number. More than $90 \%$ of the examined sequences were accurately inferred with regard to the $16 \mathrm{~S}$ rRNA gene copy number within a twofold error. Strains for which the inferred 16S rRNA gene copy number differed from the real copy number by more than threefold were phylogenetically less-studied taxa or taxa that have very different copy numbers compared to closely related strains (Additional file 6).

Figure 6 presents the two theoretical genus compositions (i and ii) and two VITCOMIC2-based genus compositions (iii and iv) of mock metagenomic sequencing data from Singer et al. [37]. We removed the Thermobacillus abundances data from all compositions, as the authors reported that there was contamination by Thermobacillus. VITCOMIC2-based genus compositions contain "Others" (other genera), which correspond to genera that did not exist in the original mock community. Because almost all of the genera in "Others" are closely related to the 23 genera in the mock community, "Others" may be artifacts that were produced from the combination of sequencing errors in metagenomic shotgun reads and misassignments of genera by VITCOMIC2. The Pearson correlation coefficients was 0.79 between (ii) the theoretical genus composition (based on multiplying the molarity of the DNA of each species and the 16S rRNA gene copy number of the genome) and (iii) the 16S rRNA gene sequence-based genus composition (calculated by VITCOMIC2); in comparison, the Pearson correlation coefficient was 0.59 between (i) the theoretical genus composition (based on the molarity of the DNA of each species) and (iv) the 16S rRNA gene sequence-based genus composition (calculated by VITCOMIC2 with the 16S rRNA gene copy number normalization). These Pearson correlation coefficients suggest the validity of the VITCOMIC2 method of genus assignment and 16S rRNA gene copy number normalization.

Additional file 7 presents a comparison result of genus composition inference accuracies of the Singer et al. mock community sequencing data among four tools. Spearman correlation coefficients of genus compositions between

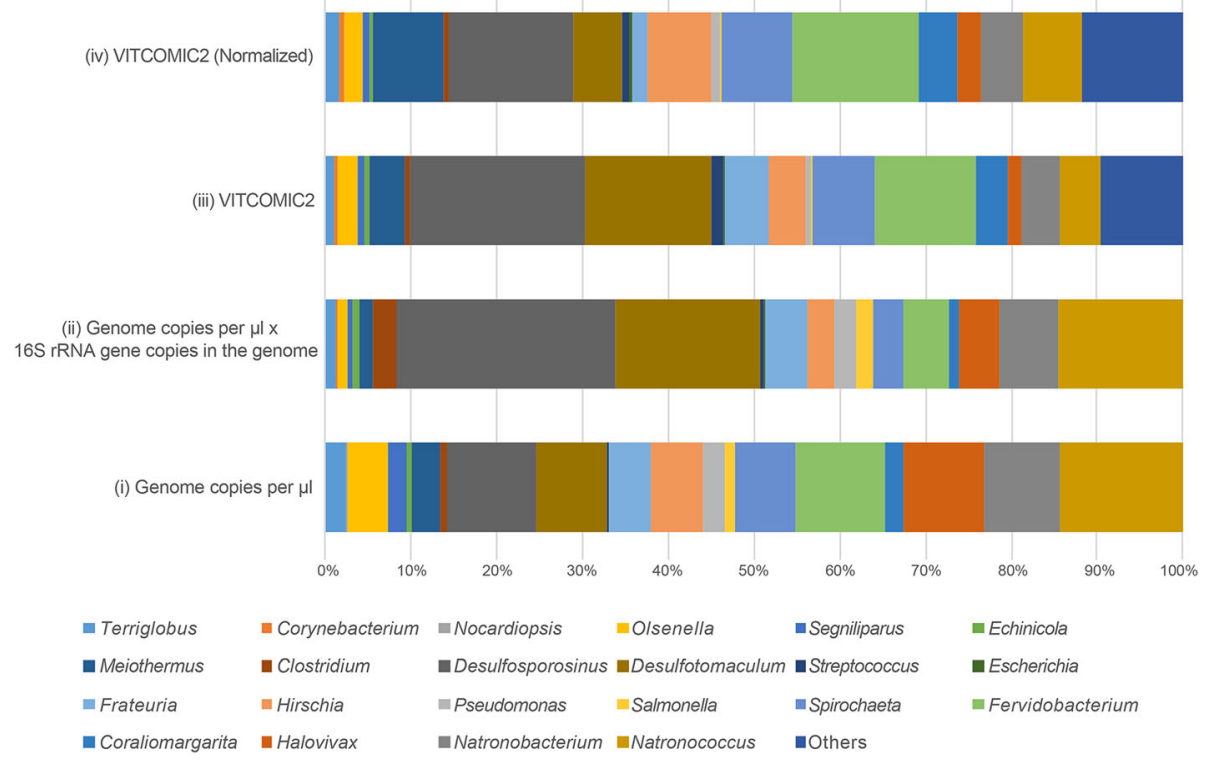

Fig. 6 Comparison of genus composition of a mock community among four samples. VITCOMIC2 (Normalized) indicates the 16S rRNA gene sequence-based genus composition as calculated by VITCOMIC2 after conducted the 16S rRNA gene copy number normalization. (i), (ii), (iii), and (iv) correspond to sample descriptions in the main text: Implementation Section: "Validation of accuracy of the genus assignments and the 165 rRNA gene copy number normalization by using mock community metagenomic data" 
the theoretical composition and each tool's inferred composition are described. Except for RiboTagger, because three tools used same reference sequence database in this analysis, the Spearman correlation coefficient differences indicates the genus inference accuracy differences caused by algorithmic differences among three tools. Among four tools, VITCOMIC2 exhibits highest genus composition inference accuracy. This comparison result also suggests that the validity of the VITCOMIC2 method of genus assignment.

\section{Validation of specificity of VITCOMIC2 with metagenomic shotgun sequencing data}

Additional file 8 presents the results of the specificity analysis of the VITCOMIC2 16S rRNA gene sequence extraction method as compared with an alternative sequence extraction method using BLAST + and the original RDP sequence database. Although the latter method extracted more sequences than did the VITCOMIC2 method, most of the specifically extracted sequences in the two methods were not $16 \mathrm{~S}$ rRNA gene sequences (e.g., they included tRNA genes, ITS regions, and $23 \mathrm{~S}$ rRNA genes). Because the evolutionary histories differ between 16S rRNA genes and other genes/regions, such non-16S rRNA gene sequences may confound the analysis of microbial community composition and diversity. Although some of the 16S rRNA gene sequences were specifically obtained with the method using the original RDP database, these sequences represented only a small percentage $(0.4 \%)$ compared with that of false positive sequences $(5.6 \%)$ that were specifically identified with the original RDP database. In addition, the calculation time needed for the sequence extraction method using the BLAST+ and original RDP sequence database was $10 \mathrm{~h} 14 \mathrm{~min}$ using a computer with four CPU cores. By contrast, the corresponding calculation time for VITCOMIC2 was $82 \mathrm{~min}$. This result indicates that VITCOMIC2 specifically and rapidly extracts $16 \mathrm{~S}$ rRNA gene sequences from metagenomic shotgun sequencing data.

\section{Species inference based on the nearly full-length $16 \mathrm{~S}$ rRNA gene sequence reconstructed from metagenomic shotgun sequencing data}

Additional file 9 presents the results of reconstruction and species assignment of the nearly full-length $16 \mathrm{~S}$ rRNA gene sequence reconstructed from mock metagenomic sequencing data [37]. All five sequences showed $100 \%$ sequence identity with the original $16 \mathrm{~S}$ rRNA gene sequence of the strain. Because this reconstruction method does not conduct the reference-based mapping assembly, some of the sequences contained ITS and intergenic regions located near the $5^{\prime}$ and $3^{\prime}$ ends of the $16 \mathrm{~S}$ rRNA gene. Although it is difficult to know why only the $16 \mathrm{~S}$ rRNA gene sequences from four minor species were able to be reconstructed, one possibility is that the Illumina sequencing platform-dependent sequence errors in some specific sequence patterns [46] may make assembly difficult in the $16 \mathrm{~S}$ rRNA gene sequences of major (i.e., high read coverage) species.

\section{Conclusions}

We developed VITCOMIC2 as a means to rapidly analyze the phylogenetic composition of microbial communities based on data from the 16S rRNA gene sequences obtained from both metagenomic shotgun and amplicon sequencing with normalization of $16 \mathrm{~S}$ rRNA gene copy number differences among genomes. The results from each VITCOMIC2 analysis are graphically visualized with two levels of taxonomic rank to facilitate understanding of taxonomic features at the level of the whole community and of the specific taxonomic groups. In addition to phylogenetic composition analyses, the combination of a highly accurate reference 16S rRNA gene sequence database in VITCOMIC2 and a rapid sequence identity search by CLASTV made it possible to reconstruct nearly full-length $16 \mathrm{~S}$ rRNA gene sequences and assign species from metagenomic shotgun sequencing data. This concise and rapid analysis method with the interactive visualization system of VITCOMIC2 will undoubtedly contribute to an intuitive understanding of the phylogenetic composition of microbial communities for diverse users.

\section{Additional files}

Additional file 1: Length distribution of 165 rRNA gene sequences in the RDP database. The length distribution of 1,345,732 16S rRNA gene sequences in the RDP database is shown after trimming of the $5^{\prime}$ and $3^{\prime}$ ends. (PDF $815 \mathrm{~kb}$ )

Additional file 2: List of RDP IDs for the 28,977 high-quality $16 \mathrm{~S}$ rRNA gene sequences in VITCOMIC2. (XLS $3610 \mathrm{~kb}$ )

Additional file 3: List of phylum names for the 28,977 high-quality $16 \mathrm{~S}$ rRNA gene sequences in VITCOMIC2. (XLS $21 \mathrm{~kb}$ )

Additional file 4: List of strain names in the 16S rRNA gene copy number reference database. a. For each strain, a RefSeq ID is shown of the representative replicon that contains 165 rRNA gene copies. b. Class name of the strain in the phylum Proteobacteria or phylum name of the strain in other phyla is shown. (XLS $210 \mathrm{~kb}$ )

Additional file 5: Distribution of the leave-one-out accuracy validation for estimating 16S rRNA gene copy number with the VITCOMIC2 copy number normalization method. The value 0 on the horizontal axis indicates the number of species that can be estimated based on the correct 16S rRNA gene copy number by applying the VITCOMIC2 copy number normalization method. (PNG $83 \mathrm{~kb}$ )

Additional file 6: List of the strains for which the inferred 16S rRNA gene copy number differed by more than threefold as compared with the real copy number. (DOC 24 kb)

Additional file 7: Correlation coefficients of genus composition of the Singer et al. mock community between theoretical composition and four tools' composition. (XLS 63 kb)

Additional file 8: Number of assigned sequences for several sequence categories using the two different reference sequence databases. a. 
"Others" indicates that sequences contain more than two categories of sequences (e.g., 165 rRNA genes and tRNA genes). (XLS 25 kb)

Additional file 9: Species assignment results from reconstructed nearly full-length 165 rRNA gene sequences from the metagenomic shotgun sequencing data of a mock community. (XLS $61 \mathrm{~kb}$ )

\section{Abbreviations}

16S rRNA-CN DB: 165 rRNA gene copy number reference database: CLAST: CUDA implemented large-scale alignment search tool; CLASTV: CLAST for VITCOMIC2; GPU: Graphics Processing Unit; ITS: internal transcribed spacer.; RDP: Ribosomal Database Project; VITCOMIC: VIsualization tool for Taxonomic COmpositions of Mlcrobial Community

\section{Acknowledgements}

Not applicable.

\section{Funding}

This study was supported through a Grant-in-Aid for Scientific Research on Innovative Areas "Genome Science", a Grant-in-Aid for Young Scientists (B) 24770015, and a Grant-in-Aid for Scientific Research (C) 15 K07214 from the Ministry of Education, Culture, Sports, Science and Technology of Japan. The publication of this article was funded by the Grant-in-Aid for Scientific Research (C) 15 K07214.

\section{Availability of data and materials}

All data generated or analyzed during this study are included in this article and its supplementary information files.

\section{Availability and requirements}

- Project name: VITCOMIC2.

- Project home page: http://vitcomic.org/.

- Archived version: version 2.0.

- Operating system(s): Platform independent.

- Programming language: JavaScript, Perl, Bash, CUDA.

- Other requirements: NVIDIA Fermi/Kepler architecture GPU, CUDA 7.0

- License: GNU GPL.

- Any restrictions to use by non-academics: None.

\section{Authors' contributions}

$\mathrm{HM}, \mathrm{TM}$, and KK designed the study. HM, TM, and MY developed the methods and performed the analyses. TY and KK provided advice on the analyses. HM, TM, and MY drafted the manuscript, and TY and KK critically revised the manuscript. All authors read and approved the final manuscript.

\section{Ethics approval and consent to participate}

Not applicable.

\section{Consent for publication}

Not applicable.

\section{Competing interests}

The authors declare that they have no competing interests.

\section{Publisher's Note}

Springer Nature remains neutral with regard to jurisdictional claims in published maps and institutional affiliations.

\section{Author details}

'Genome Evolution Laboratory, Center for Information Biology, National Institute of Genetics, Mishima 411-8540, Japan. ${ }^{2}$ Department of Biological Information, Graduate School of Bioscience and Biotechnology, Tokyo Institute of Technology, 2-12-1 M6-3, Ookayama, Meguro-ku, Tokyo 152-8550, Japan

\section{Published: 19 March 2018}

\section{References}

1. Pace NR. A molecular view of microbial diversity and the biosphere. Science. 1997;276:734-40.
2. Hamady M, Knight R. Microbial community profiling for human microbiome projects: tools, techniques, and challenges. Genome Res. 2009;19:1141-52.

3. Ludwig W, Strunk O, Westram R, Richter L, Meier H, Yadhukumar BA, Lai T, Steppi S, Jobb G, Förster W, Brettske I, Gerber S, Ginhart AW, Gross O, Grumann S, Hermann S, Jost R, König A, Liss T, Lüssmann R, May M, Nonhoff B, Reichel B, Strehlow R, Stamatakis A, Stuckmann N, Vilbig A, Lenke M, Ludwig T, Bode A, Schleifer KH. ARB: a software environment for sequence data. Nucleic Acids Res. 2004;32:1363-71.

4. Letunic I, Bork P. Interactive tree of life (iTOL): an online tool for phylogenetic tree display and annotation. Bioinformatics. 2007;23:127-8.

5. Sun Y, Cai Y, Liu L, Yu F, Farrell ML, McKendree W, Farmerie W. ESPRIT: estimating species richness using large collections of 165 rRNA pyrosequences. Nucleic Acids Res. 2009;37:e76.

6. Kemena $C$, Notredame C. Upcoming challenges for multiple sequence alignment methods in the high-throughput era. Bioinformatics. 2009;25: 2455-65.

7. Mori H, Maruyama F, Kurokawa K. VITCOMIC: visualization tool for taxonomic compositions of microbial communities based on 165 rRNA gene sequences. BMC Bioinformatics. 2010;11:332.

8. VITCOMIC version 1 web site. http://vitcomic1.vitcomic.org/ Accessed 29 June 2017.

9. Somboonna N, Assawamakin A, Wilantho A, Tangphatsornruang S, Tongsima S. Metagenomic profiles of free-living archaea, bacteria and small eukaryotes in coastal areas of Sichang island. Thailand BMC Genomics. 2012; 13:S29.

10. Sundarakrishnan B, Pushpanathan M, Jayashree S, Rajendhran J, Sakthivel N, Jayachandran S, Gunasekaran P. Assessment of microbial richness in pelagic sediment of Andaman sea by bacterial tag encoded FLX titanium amplicon pyrosequencing (bTEFAP). Indian J Microbiol. 2012;52:544-50.

11. Kato S, Nakawake M, Kita J, Yamanaka T, Utsumi M, Okamura K, Ishibashi J, Ohkuma M, Yamagishi A. Characteristics of microbial communities in crustal fluids in a deep-sea hydrothermal field of the suiyo seamount. Front Microbiol. 2013;4:85

12. Cole JR, Wang Q, Fish JA, Chai B, McGarrell DM, Sun Y, Brown $\subset$, PorrasAlfaro A, Kuske CR, Tiedje JM. Ribosomal database project: data and tools for high throughput rRNA analysis. Nucleic Acids Res. 2014;42:D633-42.

13. Acinas SG, Marcelino LA, Klepac-Ceraj V, Polz MF. Divergence and redundancy of 165 rRNA sequences in genomes with multiple rrn operons. J Bacteriol. 2004;186:2629-35.

14. Angly FE, Dennis PG, Skarshewski A, Vanwonterghem I, Hugenholtz P, Tyson GW. CopyRighter: a rapid tool for improving the accuracy of microbial community profiles through lineage-specific gene copy number correction. Microbiome. 2014;2:11.

15. Hamady M, Walker JJ, Harris JK, Gold NJ, Knight R. Error-correcting barcoded primers for pyrosequencing hundreds of samples in multiplex. Nat Methods. 2008:5:235-7.

16. Altschul SF, Gish W, Miller W, Myers EW, Lipman DJ. Basic local alignment search tool. J Mol Biol. 1990;215:403-10.

17. Hamp TJ, Jones WJ, Fodor AA. Effects of experimental choices and analysis noise on surveys of the "rare biosphere". Appl Environ Microbiol. 2009;75: 3263-70.

18. Mori H, Maruyama F, Kato H, Toyoda A, Dozono A, Ohtsubo Y, Nagata Y, Fujiyama A, Tsuda M, Kurokawa K. Design and experimental application of a novel non-degenerate universal primer set that amplifies prokaryotic 165 rRNA genes with a low possibility to amplify eukaryotic rRNA genes. DNA Res. 2014;21:217-27.

19. Camacho C, Coulouris G, Avagyan V, Ma N, Papadopoulos J, Bealer K, Madden TL. BLAST+: architecture and applications. BMC Bioinformatics. 2009;10:421.

20. VITCOMIC1 reference $16 \mathrm{~S}$ rRNA gene sequence database. http://vitcomic1. vitcomic.org/ Accessed 29 June 2017

21. Liu WT, Marsh TL, Cheng H, Forney LJ. Characterization of microbial diversity by determining terminal restriction fragment length polymorphisms of genes encoding 16S rRNA. Appl Environ Microbiol. 1997;63:4516-22.

22. Shine J, Dalgarno L. The 3 ' -terminal sequence of Escherichia coli $16 \mathrm{~S}$ ribosomal RNA: complementarity to nonsense triplets and ribosome binding sites. Proc Natl Acad Sci U S A. 1974;71:1342-6.

23. Edgar RC. Search and clustering orders of magnitude faster than BLAST. Bioinformatics. 2010;26:2460-1.

24. Edgar RC, Haas BJ, Clemente JC, Quince C, Knight R. UCHIME improves sensitivity and speed of chimera detection. Bioinformatics. 2011;27:2194-200. 
25. Reference gold database. http://drive5.com/uchime/gold.fa Accessed 29 June 2017.

26. Kloesges T, Popa O, Martin W, Dagan T. Networks of gene sharing among 329 Proteobacterial genomes reveal differences in lateral gene transfer frequency at different phylogenetic depths. Mol Biol Evol. 2011;28:1057-74.

27. Katoh K, Standley DM. MAFFT. Iterative refinement and additional methods. Methods Mol Biol. 2014;1079:131-46.

28. Felsenstein J. PHYLIP-phylogeny inference package (version 3.2). Cladistics. 1989;5:164-6.

29. Kimura M. A simple method for estimating evolutionary rates of base substitutions through comparative studies of nucleotide sequences. J Mol Evol. 1980;16:111-20

30. Yano M, Mori H, Akiyama Y, Yamada T, Kurokawa K. CLAST: CUDA implemented large-scale alignment search tool. BMC Bioinformatics. 2014;15:406.

31. Turnbaugh PJ, Hamady M, Yatsunenko T, Cantarel BL, Duncan A, Ley RE, Sogin ML, Jones WJ, Roe BA, Affourtit JP, Egholm M, Henrissat B, Heath AC, Knight $\mathrm{R}$, Gordon $\mathrm{Jl}$. A core gut microbiome in obese and lean twins. Nature. 2009;457:480-4.

32. Kembel SW, Wu M, Eisen JA, Green JA. Incorporating 165 gene copy number information improves estimates of microbial diversity and abundance. PLoS Comput Biol. 2012;8:e1002743.

33. Rastogi R, Wu M, Dasqupta I, Fox GE. Visualization of ribosomal RNA operon copy number distribution. BMC Microbiol. 2009;9:208.

34. Lagesen K, Hallin P, Rødland EA, Staerfeldt HH, Rognes T, Ussery DW. RNAmmer: consistent and rapid annotation of ribosomal RNA genes. Nucleic Acids Res. 2007:35:3100-8.

35. NCBI Genome Database. ftp://ftp.ncbi.nih.gov/genomes/genbank/bacteria/ Accessed 29 June 2017.

36. Resource Coordinators NCBI. Database resources of the National Center for biotechnology information. Nucleic Acids Res. 2015;43:D6-D17.

37. Singer E, Andreopoulos B, Bowers RM, Lee J, Deshpande S, Chiniguy J, Ciobanu D, Klenk HP, Zane M, Daum C, Clum A, Cheng JF, Copeland A, Woyke T. Next generation sequencing data of a defined microbial mock community. Sci Data. 2016;3:160081

38. Rodrigues JFM, Schmidt TSB, Tackmann J, von Mering C. MAPseq: highly efficient k-mer search with confidence estimates, for rRNA sequence analysis. Bioinformatics. 2017:33:3808-10.

39. Kopylova E, Noe L, Touzet H. SortMeRNA: fast and accurate filtering of ribosomal RNAs in metatranscriptomic data. Bioinformatics. 2012;28:3211-7.

40. Xie C, Goi CL, Huson DH, Little PF, Williams RB. RiboTagger: fast and unbiased 16S/18S profiling using whole community shotgun metagenomic or metatranscriptome surveys. BMC Bioinformatics. 2016;17:508.

41. Clarridge JE 3rd. Impact of $165 \mathrm{rRNA}$ gene sequence analysis for identification of bacteria on clinical microbiology and infectious diseases. Clin Microbiol Rev. 2004;17:840-62.

42. Wang Q, Garrity GM, Tiedje JM, Cole JR. Naiive Bayesian classifier for rapid assignment of rRNA sequences into the new bacterial taxonomy. Appl Environ Microbiol. 2007:73:5261-7.

43. VITCOMIC2 web server. http://vitcomic.org/vitcomic2/ Accessed 29 June 2017.

44. Cannone JJ, Subramanian S, Schnare MN, Collett JR, D'Souza LM, Du Y, Feng B, Lin N, Madabusi LV, Müller KM, Pande N, Shang Z, Yu N, Gutell RR. The comparative RNA web (CRW) site: an online database of comparative sequence and structure information for ribosomal, intron, and other RNAs. BMC Bioinformatics. 2002;3:2.

45. VITCOMIC2 Comparison web server. http://vitcomic.org/ Accessed 29 June 2017.

46. Nakamura K, Oshima T, Morimoto T, Ikeda S, Yoshikawa H, Shiwa Y, Ishikawa S, Linak MC, Hirai A, Takahashi H, Altaf-Ul-Amin M, Ogasawara N, Kanaya S. Sequence-specific error profile of Illumina sequencers. Nucleic Acids Res. 2011;39:e90

\section{Submit your next manuscript to BioMed Central and we will help you at every step:}

- We accept pre-submission inquiries

- Our selector tool helps you to find the most relevant journal

- We provide round the clock customer support

- Convenient online submission

- Thorough peer review

- Inclusion in PubMed and all major indexing services

- Maximum visibility for your research

Submit your manuscript at www.biomedcentral.com/submit

) Biomed Central 